\title{
Policonsumo de drogas y su relación con el contexto familiar y social en jóvenes universitarios
}

\section{Polydrug use and its relationship with the familiar and social context amongst young college students}

\author{
Olga Hernández-Serrano*, Sílvia Font-Mayolas**, Maria Eugènia Gras**
}

*Escola Universitària de la Salut i l'Esport. Universitat de Girona, Girona

**Instituto de Investigación sobre Calidad de Vida. Universitat de Girona, Girona

\section{Resumen}

El policonsumo de drogas es cada vez más prevalente entre los jóvenes españoles. El Observatorio Europeo de las Drogas y las Toxicomanías establece tres tipologías de policonsumo: Patrón A (consumidores de alcohol y tabaco), Patrón B (consumidores de cannabis junto con alcohol y/o tabaco) y Patrón C (consumidores de cannabis junto con alcohol y tabaco y al menos otra droga ilegal). Los objetivos son: 1) estudiar la frecuencia de consumo de drogas en una muestra de jóvenes universitarios españoles del ámbito de la salud y el deporte según el sexo; 2) describir los patrones de policonsumo; 3) estudiar la relación entre el policonsumo de los participantes y el policonsumo de las personas de su entorno próximo (padres, hermanos, pareja y mejor amigo). La muestra consta de 480 universitarios ( $43.7 \%$ chicas) entre 18 y 36 años. Se administró un autoinforme para evaluar el policonsumo de los participantes y de sus referentes más próximos. Un $46 \%$ de los participantes eran consumidores de dos o más sustancias, de los cuales un $29.4 \%$ correspondían al Patrón A, un 50.7\% al Patrón B, un $16.7 \%$ al Patrón C y un $3.2 \%$ a otros patrones de policonsumo (alcohol + cocaína; alcohol + cocaína + tabaco; alcohol + inhalantes; anfetaminas + alucinógenos + Spice). Se observa una elevada concordancia entre el policonsumo de los participantes y el de sus referentes próximos, de modo que si el referente es policonsumidor es más probable que el participante también lo sea. El policonsumo de drogas en el entorno próximo de los jóvenes deviene uno de los elementos clave a tener en cuenta en futuras campañas preventivas. Palabras clave: policonsumo de drogas, factores contextuales, estudiantes universitarios.

\begin{abstract}
The prevalence of polydrug use continues to grow among Spanish college students. The European Observatory for Drugs and Addictions establishes three different types of polydrug use: Pattern A (consumers of alcohol and tobacco), Pattern B (consumers of cannabis plus alcohol and/or tobacco) and Pattern C (consumers of cannabis plus alcohol plus tobacco plus at least one other kind of illegal drug). The objectives are: 1) to study the frequency of substance consumption among a sample of young Spanish undergradudates studying health and sports science according to their sex; 2) to describe the patterns of polydrug use; 3 ) to study the relationship between the polydrug use of the participants and polydrug use within their closest environment (parents, sisters or brothers, best friend and partner). The sample was composed of 480 Spanish undergraduates ( $43.7 \%$ females) aged 18 to 36 . The level of drug consumption of students and their closest reference persons was evaluated by means of a self-report measure. A total of $46 \%$ of the participants reported consumption of two or more substances; among them $29.4 \%$ corresponded to Pattern A, $50.7 \%$ to Pattern B and $16.7 \%$ to Pattern C, while $3.2 \%$ corresponded to other multiple consumption patterns (alcohol + cocaine; alcohol + cocaine + tobacco; alcohol + inhalants; amphetamines + hallucinogens + Spice). An important correlation was observed concerning polydrug use between participants and their closest reference persons: the more the reference person is a multiple consumer, the more the participant tends to consume. Polydrug use within the closest environment emerges as one of the key elements to be taken into account in further prevention programs.

Keywords: polydrug use, contextual factors, college students.
\end{abstract}


S egún el European Monitoring Centre for Drugs and Drug Addiction (EMCDDA, 2011) el policonsumo se ha convertido en una de las pautas dominantes del consumo de sustancias en Europa. Desde este observatorio las principales tipologías de policonsumo en población adolescente se clasifican en el Patrón A (consumidores de alcohol y tabaco), el Patrón B (consumidores de cannabis junto con alcohol y/o tabaco) y el Patrón C (consumidores de cannabis junto con alcohol y tabaco y al menos otra droga ilegal). Entre los jóvenes adultos de 15 a 34 años, las sustancias más extendidas son alcohol y tabaco, seguido de cannabis en todos los países europeos. A pesar de que el EMCDDA (2009a) no establece tipologías concretas de policonsumo centradas en población joven, se halla relación entre el consumo frecuente o abusivo de alcohol y una alta probabilidad de consumir cannabis o cocaína en comparación con la población general. A su vez, muchos de los consumidores de cocaína son también consumidores de cannabis. Sin embargo, la mayoría de consumidores de cannabis no son consumidores de drogas ilícitas.

Según el Observatorio Español de la Droga y las Toxicomanías (OEDT, 2011), al igual que en Europa, las sustancias de mayor consumo en población de 15 a 64 años son alcohol, tabaco y cannabis. Durante el último año, el $49.3 \%$ de los consumidores de sustancias psicoactivas admite consumir dos o más drogas. Entre los consumidores de dos sustancias, alcohol y tabaco son las drogas de mayor consumo. Entre los consumidores de tres sustancias, alcohol, tabaco y cannabis son las más extendidas. Sin embargo, los policonsumidores de cuatro o más sustancias toman en mayor medida alcohol, tabaco, cannabis, cocaína en polvo y éxtasis/drogas de diseño (OEDT, 2011). En este sentido, y específicamente en población universitaria, determinados estudios han explorado si el uso de una sustancia incrementa la probabilidad de consumir otras drogas encontrando una asociación positiva en función del tipo de sustancia. Así pues, se halla relación entre el consumo en alguna ocasión de éxtasis y el consumo de marihuana, siendo más probable el uso de otras sustancias como cocaína, heroína, LSD e inhalantes entre aquellos consumidores de éxtasis que de marihuana (Wish, Fitzelle, O’ Grady, Hsu y Arria, 2006). Otros trabajos encuentran una fuerte asociación entre el consumo de tabaco y el consumo de cannabis: nueve de cada diez jóvenes que nunca habían fumado y sólo 1.5 de cada diez fumadores tampoco habían consumido nunca cannabis (Font-Mayolas, Gras y Planes, 2006). En la misma línea, en un estudio sobre consumo de cocaína se observó que los jóvenes que habitualmente consumen cocaína son también mayoritariamente consumidores de alcohol y cannabis (Patiño-Masó, Gras-Pérez, Font-Mayolas y Baltasar-Bagué, 2013). Asimismo, Tirado, Aguaded y Marin (2009) indican que el policonsumo (alcohol de baja graduación + tabaco) es el principal factor de riesgo del consumo de alcohol de alta graduación en universitarios.
A nivel demográfico, en los últimos años se han investigado las diferencias según el sexo y la edad de los jóvenes policonsumidores de drogas. Muchos de estos estudios indican que los hombres presentan un mayor policonsumo que las mujeres (Substance Abuse and Mental Health Services Administration, 2009; Whitehorne-Smith et al., 2012). Respecto a la edad, datos en adultos jóvenes confirman relaciones con el policonsumo de sustancias (Ramo, Liu y Prochaska, 2012). Además, el policonsumo entre los jóvenes adultos se asocia a graves problemas como bajo desempeño a nivel cognitivo, problemas físicos, accidentes de tráfico, lesiones, enfermedades infecciosas y de transmisión sexual, suicidios, peleas y otros actos de violencia, además de poder llegar a convertirse en un patrón más permanente de consumo de varias sustancias (Connor, Gullo, White y Kelly, 2014; EMCDDA, 2011, 2014; Halley, Forster, Wood, Baezconde-Garbanati y Beth, 2014; Hughes et al., 2009; Mariño, Castro y Torrado, 2012; Trenz et al., 2012).

Comprender los factores de riesgo y protección asociados al policonsumo de sustancias se hace necesario para el desarrollo de intervenciones dirigidas a la prevención de estos problemas. En este sentido, merece especial atención la revisión de Stone, Becker, Huber y Catalano (2012) sobre factores de riesgo y protección en la edad adulta temprana (entre 18 y 26 años). Estos autores, de acuerdo con Hawkins, Catalano y Miller (1992), establecen una clasificación en factores contextuales (sexo, etnia, indicadores biológicos, indicadores prenatales y postnatales, nivel socioeconómico, nivel educativo de los padres, estatus de los padres, historia familiar de consumo de sustancias, problemas psicopatológicos de los padres, barrios desfavorecidos, normas sociales, leyes e impuestos, disponibilidad) e interpersonales (historia pasada de abuso de sustancias, relación familiar, gestión familiar, atribuciones externas e internas, consumo de drogas y expectativas en la adolescencia, actitudes favorables y expectativas, situación en la vida, estatus/nivel laboral, asistencia a la universidad, relación con el grupo de iguales, creencias morales, participaciones religiosas, factores educativos, estado civil casado o comprometido en pareja, eventos estresantes). El presente estudio se centra en los factores contextuales y en concreto en el consumo por parte de los miembros del entorno más próximo al sujeto como posible factor de riesgo del policonsumo de drogas.

Desde la teoría del aprendizaje social propuesta por Bandura y Walters (1979), el aprendizaje puede explicarse a través de la observación de otros modelos de conducta o modelado, que puede propiciar el inicio al consumo de sustancias en los jóvenes y aumentar su frecuencia. Existen evidencias sobre la relación entre el consumo de determinadas sustancias en familiares, pareja y amigos, y la conducta de consumo en jóvenes universitarios. Por ejemplo, Font-Mayolas y Planes (2000) hallaron que los jóvenes fumadores solían tener padre y/o madre y/o amigos también fumadores mientras que en el caso de la pareja y los hermanos no en- 
contraron diferencias. Sin embargo, la mayor parte de estas investigaciones se centran en el estudio independiente de sustancias como alcohol, tabaco, marihuana o medicamentos (Becoña et al., 2012; Buu et al., 2009; Martín-Montañez et al., 2011; Mason et al. 2009), siendo escasos los trabajos que buscan relaciones entre policonsumo de drogas y estos factores contextuales. En relación al policonsumo de los amigos, los resultados encontrados coinciden en apoyar una asociación positiva entre el consumo de varias sustancias y tener amigos policonsumidores. Por ejemplo, Varela, Salazar, Cáceres y Tovar (2007) hallaron una fuerte asociación entre el consumo de marihuana, opiáceos, cocaína y éxtasis en estudiantes universitarios brasileños, y mantener una relación de amistad con otros que también consumen drogas pudiendo aumentar dicha práctica de consumo. En otros estudios, la iniciación al consumo de cannabis, además de asociarse al consumo previo de alcohol y tabaco, se relacionó principalmente con formar parte de un grupo de amigos que consumen drogas (Vázquez y Becoña, 2000). En relación a la forma en que los estudiantes aprenden sobre el policonsumo de sustancias psicoactivas, un alto porcentaje de estudiantes universitarios afirma que la vía más usada es a través de un amigo (Herrera et al., 2012; Riquelme et al., 2012; Veloza et al., 2012). Recientemente, se encuentra que tener tres o cuatro amigos cercanos consumidores de sustancias es un predictor relevante de problemas de consumo de drogas en comparación con aquellos jóvenes con amigos no consumidores (Halley et al., 2014). Respecto a los datos en relación al consumo de más de una sustancia en los hijos y el policonsumo de los padres, los resultados no son concluyentes. Algunos autores indican que el consumo de alcohol y tabaco de un $12 \%$ de los estudiantes universitarios se relaciona con el aprendizaje por medio de un miembro de la familia como es el padre, la madre, algún/os hermano/os u otros familiares (Veloza et al., 2012). Sin embargo, otros autores encuentran que tener un padre o una madre consumidor de drogas no es un predictor relevante en el desarrollo de problemas de consumo de los hijos en la juventud (Halley et al., 2014). En el caso de la pareja, Veloza et al. (2012) detectan asociación entre el policonsumo de alcohol y tabaco en estudiantes universitarios y tener novio/a policonsumidor en un $13 \%$ del total de la muestra estudiada.

En general, la mayor parte de los estudios sobre policonsumo de drogas pertenecen a la población adolescente (Connell, Gilreath, Aklin y Brex, 2010; Font-Mayolas et al., 2013; Martínez, Fuentes, García y Madrid, 2013). En población universitaria, muchos trabajos focalizan su atención en una única sustancia (ej. Font-Mayolas et al., 2006) y aquellos que se centran en el consumo de varias drogas evalúan un número limitado de sustancias (principalmente tabaco, alcohol, cannabis, cocaína, éxtasis y medicamentos). Además, los datos disponibles sobre policonsumo de drogas incluyen estudios pertenecientes al área social, humanidades o salud realizados en contextos geográficos y socioculturales diferentes al ámbito español (Gómez, Herde, Laffee, Lobo y Martín, 2007; Herrera et al., 2012; Prieto et al., 2012; Veloza et al., 2012; Whitehorne-Smith et al., 2012). En este sentido, diversos autores destacan la importancia de estudiar la población universitaria del área de la salud, pero también del deporte, por ser consideradas una población vulnerable (Lores, Murcia, Gutiérrez y Sicilia, 2003; Oliveira y Furegato, 2008; Urrego, 2002) a cargo del cuidado de la salud, el grado de bienestar y el nivel de calidad de vida de la población. Además, una característica novedosa de los mercados europeos es la disponibilidad creciente de «nuevas sustancias psicotrópicas» no controladas por los tratados internacionales de control de drogas (EMCDDA, 2014). Sustancias como el Spice (marihuana sintética), incluida en el repertorio de drogas dentro de este estudio, es muy popular entre los jóvenes, ocupando el segundo lugar después de la marihuana entre las drogas ilícitas más consumidas (EMCDDA, 2009b). Por lo tanto, se destaca la importancia de conocer la problemática del policonsumo de drogas teniendo en cuenta sustancias de nueva generación. Así, los objetivos del presente estudio son: 1) estudiar la frecuencia de consumo de drogas en una muestra de jóvenes universitarios españoles del ámbito de la salud y el deporte según el sexo; 2) describir los patrones de policonsumo de estos jóvenes y; 3) estudiar la asociación del uso de más de una sustancia con el policonsumo de las personas de su entorno más próximo (padre o tutor, madre o tutora, hermanos/as, mejor amigo/a, y la pareja).

\section{Método}

\section{Participantes}

La población diana son estudiantes de una escuela adscrita a la Universidad de Girona (Escola Universitària de la Salut i l'Esport, EUSES) con Campus en Salt (Girona). Se llevó a cabo un estudio descriptivo de prevalencia y de asociación cruzada. De un total de 804 estudiantes matriculados, 324 no asistieron a clase el día y la hora de la recogida de información. Por tanto, el porcentaje de participación es del $59.7 \%$ no estando asociado a la falta de interés por parte del alumnado. Forman parte de la muestra el $100 \%$ de los estudiantes que estaban presentes el día de la recogida de datos. Así, la muestra total está formada por 480 estudiantes universitarios de los que el $43.7 \%$ son mujeres y el $56.3 \%$ hombres con edades entre los 18 y 36 años $(M=21.3 ; D T$ $=2.8$ ) de primer curso $(32.5 \%)$, segundo curso $(29.3 \%)$ y tercer curso $(38.2 \%)$.

\section{Instrumentos}

Cuestionario Sociodemográfico. Evalúa información sobre el tipo de estudios, curso, edad, sexo, ocupación y rendimiento académico.

La información referente al policonsumo de drogas se recogió a través de un autoinforme mediante el cual se evaluaron las variables que se enumeran a continuación: 
Frecuencia de consumo de los participantes. Está compuesto por 9 ítems de alternativa múltiple ("nunca", "ocasionalmente”, "una vez por semana”, "más de una vez por semana", "cada día”) que evalúan la frecuencia del consumo de tabaco, alcohol, cannabis, cocaína, heroína, inhalantes volátiles, speed o anfetaminas, alucinógenos y Spice.

Frecuencia de consumo de las personas de su entorno. Está compuesto por 9 ítems de alternativa múltiple ("no lo sé/ no procede”, "nunca”, “ocasionalmente”, "una vez por semana”, "más de una vez por semana”, "cada día”) que evalúan la creencia que tiene el estudiante sobre la frecuencia del consumo (tabaco, alcohol, cannabis, cocaína, heroína, inhalantes volátiles, speed o anfetaminas, alucinógenos y Spice) del padre o tutor, la madre o tutora, algún hermano/a, el mejor amigo/a, y la pareja.

La consistencia interna del instrumento evaluada a partir del alfa de Cronbach es de 0.81 .

\section{Procedimiento}

La recogida de datos se realizó en los tres primeros cursos de los grados de Fisioterapia y Ciencias de la Actividad Física y el Deporte. El presente estudio fue aprobado por la Comisión de Investigación de la Universidad de Girona. Previamente al inicio de la recogida de datos se llevó a cabo una entrevista con los directivos de la escuela con el objetivo de exponer las características de la investigación y solicitar la participación. Posteriormente, se contactó con los profesores correspondientes para concretar una fecha de administración del cuestionario. El autoinforme fue administrado en un único momento durante el curso escolar 2013/2014 por los responsables de la investigación. Se solicitó la colaboración voluntaria de los estudiantes, al mismo tiempo se garantizó la confidencialidad en las respuestas y su uso únicamente con fines de investigación.

\section{Resultados}

En la Tabla 1 se presenta el consumo de sustancias de los participantes según el sexo. Se observan diferentes frecuencias de consumo según la sustancia. El tabaco es la sustancia más consumida diariamente seguida del cannabis. El consumo diario de alcohol es poco frecuente entre los participantes aunque son minoría los que informan no consumir nunca esta sustancia. Sólo un joven de la muestra informa consumir cocaína y alucinógenos a diario y ningún participante informa de consumo diario de heroína, inhalantes volátiles, speed o anfetaminas y Spice.

El cannabis es la droga ilegal más consumida, tres de cada diez participantes informan consumirla con más o menos frecuencia. Entre 10 y 21

Tabla 1 participantes consumen ocasionalmente cocaína, speed/ anfetaminas, inhalantes volátiles o alucinógenos. La heroína y el Spice son las sustancias menos consumidas: sólo dos hombres informan de un uso ocasional de la heroína y otros dos del Spice (uno de ellos ocasional y el otro superior a una vez por semana).

No se observan diferencias estadísticamente significativas entre hombres y mujeres en la frecuencia de consumo de sustancias (Tabaco: $\chi_{(4)}^{2}=7.47 ; p=0.11$; Alcohol: $\chi_{(4)}^{2}=$ 1.84; $p=0.77$; Cocaina: $\chi_{(3)}^{2}=3.62 ; p=0.31$; Heroína: $\chi^{2}$ ${ }_{(1)}=1.56 ; p=0.21$; Inhalantes: $\chi_{(1)}^{2}=0.37 ; p=0.55$; Speed o anfetaminas: $\chi_{(2)}^{2}=2.27 ; p=0.32$; Alucinógenos: $\chi_{(3)}^{2}=$ $1.62 ; p=0.65$; Spice: $\left.\chi_{(2)}^{2}=1.56 ; p=0.46\right)$ excepto en el caso del cannabis $\left(\chi_{(4)}^{2}=14.07 ; p=0.007\right)$ donde se observa que significativamente más mujeres que hombres no la han probado nunca $(z=-2.24 ; p=0.025)$ y más hombres que mujeres informan de consumos superiores a una vez por semana $(z$ $=2.58 ; p=0.001)$

Un $46 \%$ de los participantes $(45.7 \%$ de los hombres y $46.4 \%$ de las mujeres) informan ser consumidores de dos o más sustancias, sin diferencias estadísticamente significativas por sexo $(z=0.14 ; p=0.88)$. Entre los policonsumidores un $29.4 \%$ ( $20.3 \%$ de los hombres y $41.2 \%$ de las mujeres)

Frecuencia (porcentaje) de consumo de sustancias según el sexo.

\begin{tabular}{|c|c|c|c|c|c|c|}
\hline & & Nunca & $\begin{array}{l}\text { Consumo } \\
\text { ocasional }\end{array}$ & $\begin{array}{c}\text { Una vez } \\
\text { por } \\
\text { semana }\end{array}$ & $\begin{array}{c}\text { Más de } \\
1 \text { vez/ } \\
\text { semana }\end{array}$ & $\begin{array}{c}\text { Cada } \\
\text { día }\end{array}$ \\
\hline \multirow{3}{*}{$\begin{array}{l}\text { Tabaco } \\
(n=478)\end{array}$} & Hombres & $64.7 \%$ & $10.0 \%$ & $4.5 \%$ & $3.0 \%$ & $17.8 \%$ \\
\hline & Mujeres & $60.4 \%$ & $17.4 \%$ & $1.9 \%$ & $3.4 \%$ & $16.9 \%$ \\
\hline & Total & $62.8 \%$ & $13.2 \%$ & $3.4 \%$ & $3.2 \%$ & $17.4 \%$ \\
\hline \multirow{3}{*}{$\begin{array}{l}\text { Alcohol } \\
(n=476)\end{array}$} & Hombres & $6.7 \%$ & $43.9 \%$ & $27.5 \%$ & $20.4 \%$ & $1.5 \%$ \\
\hline & Mujeres & $5.4 \%$ & $46.8 \%$ & $28.3 \%$ & $17.1 \%$ & $2.4 \%$ \\
\hline & Total & $6.1 \%$ & $45.1 \%$ & $27.8 \%$ & $19.0 \%$ & $1.9 \%$ \\
\hline \multirow{3}{*}{$\begin{array}{l}\text { Cannabis } \\
(n=479)\end{array}$} & Hombres & $65.1 \%$ & $20.1 \%$ & $3.0 \%$ & $4.8 \%$ & $7.1 \%$ \\
\hline & Mujeres & $74.5 \%$ & $20.7 \%$ & $1.9 \%$ & $1.0 \%$ & $1.9 \%$ \\
\hline & Total & $69.2 \%$ & $20.3 \%$ & $2.5 \%$ & $3.1 \%$ & $4.8 \%$ \\
\hline \multirow{3}{*}{$\begin{array}{l}\text { Cocaína } \\
(n=479)\end{array}$} & Hombres & $93.7 \%$ & $5.6 \%$ & $0.4 \%$ & $0 \%$ & $0.4 \%$ \\
\hline & Mujeres & $97.1 \%$ & $2.9 \%$ & $0 \%$ & $0 \%$ & $0 \%$ \\
\hline & Total & $95.2 \%$ & $4.4 \%$ & $0.2 \%$ & $0 \%$ & $0.2 \%$ \\
\hline \multirow{3}{*}{$\begin{array}{l}\text { Heroína } \\
\text { ( } n=479)\end{array}$} & Hombres & $99.3 \%$ & $0.7 \%$ & $0 \%$ & $0 \%$ & $0 \%$ \\
\hline & Mujeres & $100 \%$ & $0 \%$ & $0 \%$ & $0 \%$ & $0 \%$ \\
\hline & Total & $99.6 \%$ & $0.4 \%$ & $0 \%$ & $0 \%$ & $0 \%$ \\
\hline \multirow{3}{*}{$\begin{array}{l}\text { Inhalantes } \\
\text { volátiles } \\
(n=480)\end{array}$} & Hombres & $96.7 \%$ & $3.3 \%$ & $0 \%$ & $0 \%$ & $0 \%$ \\
\hline & Mujeres & $97.6 \%$ & $2.4 \%$ & $0 \%$ & $0 \%$ & $0 \%$ \\
\hline & Total & $97.1 \%$ & $2.9 \%$ & $0 \%$ & $0 \%$ & $0 \%$ \\
\hline \multirow{3}{*}{$\begin{array}{l}\text { Speed o } \\
\text { anfetaminas } \\
\text { ( } n=480)\end{array}$} & Hombres & $95.2 \%$ & $4.5 \%$ & $0.4 \%$ & $0 \%$ & $0 \%$ \\
\hline & Mujeres & $97.6 \%$ & $2.4 \%$ & $0 \%$ & $0 \%$ & $0 \%$ \\
\hline & Total & $96.2 \%$ & $3.6 \%$ & $0.2 \%$ & $0 \%$ & $0 \%$ \\
\hline \multirow{3}{*}{$\begin{array}{l}\text { Alucinógenos } \\
(n=480)\end{array}$} & Hombres & $97 \%$ & $2.2 \%$ & $0 \%$ & $0.4 \%$ & $0.4 \%$ \\
\hline & Mujeres & $98.1 \%$ & $1.9 \%$ & $0 \%$ & $0 \%$ & $0 \%$ \\
\hline & Total & $97.5 \%$ & $2.1 \%$ & $0 \%$ & $0.2 \%$ & $0.2 \%$ \\
\hline \multirow{3}{*}{$\begin{array}{l}\text { Spice } \\
(n=480)\end{array}$} & Hombres & $99.3 \%$ & $0.4 \%$ & $0 \%$ & $0.4 \%$ & $0 \%$ \\
\hline & Mujeres & $100 \%$ & $0 \%$ & $0 \%$ & $0 \%$ & $0 \%$ \\
\hline & Total & $99.6 \%$ & $0.2 \%$ & $0 \%$ & $0.2 \%$ & $0 \%$ \\
\hline
\end{tabular}


Tabla 2

Porcentaje de participantes que consumen más de una sustancia según el policonsumo de sus referentes más próximos y resultados de la prueba ji-cuadrado.

\begin{tabular}{lccccc}
\hline & $\begin{array}{c}\text { Mejor amigo } \\
(n=\mathbf{4 6 5 )}\end{array}$ & $\begin{array}{c}\text { Padre } \\
(n=\mathbf{4 6 6 )}\end{array}$ & $\begin{array}{c}\text { Madre } \\
(n=\mathbf{4 6 7 )}\end{array}$ & $\begin{array}{c}\text { Algún hermano } \\
(n=\mathbf{4 5 3})\end{array}$ & $\begin{array}{c}\text { Pareja } \\
(n=\mathbf{3 6 1 )}\end{array}$ \\
\hline $\begin{array}{l}\text { Referente no policonsumidor } \\
\begin{array}{l}\text { Referente } \\
\text { policonsumidor }\end{array}\end{array}$ & $20.3 \%$ & $41.9 \%$ & $42.8 \%$ & $32.4 \%$ & $30.5 \%$ \\
$\begin{array}{l}\chi^{2} \\
(p)\end{array}$ & $59.9 \%$ & $58.4 \%$ & $58.1 \%$ & $68.0 \%$ & $69.5 \%$ \\
\hline
\end{tabular}

correspondían al patrón de consumo A (consumidores de alcohol y tabaco), un $50.7 \%$ (53.7\% de los hombres y $46.4 \%$ de las mujeres) al Patrón B (consumidores de cannabis junto con alcohol y/o tabaco), un $16.7 \%$ (22\% de los hombres y $10.3 \%$ de las mujeres) al Patrón C (consumidores de cannabis junto con alcohol y/o tabaco y al menos otra droga ilegal) y un $3.2 \%$ (4.1\% de los hombres y $2.1 \%$ de las mujeres) a otros patrones atípicos de policonsumo. La proporción de mujeres clasificadas en el patrón A de consumo presenta diferencias estadísticamente significativas con los hombres, siendo mayor la proporción de mujeres que la de hombres $(z=-3.18 ; p=0.0014)$ mientras que la proporción de hombres en el patrón C es significativamente superior a la de mujeres $(z=2.41 ; p=0.016)$. No se observan diferencias estadísticamente significativas en función del sexo ni en el patrón $\mathrm{B}(z=1.07 ; p=0.28)$ ni en los patrones atípicos de consumo $(z=0.87 ; p=0.38)$.

De los siete participantes (3.2\% de los policonsumidores) que informan de otros patrones de policonsumo, cuatro consumían alcohol y cocaína, uno tabaco, alcohol y cocaína, uno alcohol e inhalantes y uno anfetaminas, alucinógenos y Spice.

En la Tabla 2 se presenta el porcentaje de policonsumidores de la muestra en función del policonsumo de sus referentes más próximos: mejor amigo, padre, madre, hermanos y pareja y los resultados de la prueba ji-cuadrado. Se observa una elevada concordancia entre el policonsumo de sustancias de los participantes y el de sus referentes más próximos, de modo que si el referente es policonsumidor es más probable que el participante también lo sea.

En la Tabla 3 se presentan los resultados de la regresión logística binaria para predecir el policonsumo en función de la edad, el sexo y el policonsumo de los referentes más próximos. Cuando se analizan conjuntamente las variables que mejor predicen el uso de más de una sustancia por parte de los participantes, se observa que son las siguientes: el policonsumo de su mejor amigo, de algún hermano o de la pareja. Si el mejor amigo del participante es policonsumidor, aumenta entre 3.29 y 11.43 veces la probabilidad de que él mismo sea policonsumidor en comparación con aquellos participantes cuyo mejor amigo no es policonsumidor; si tiene algún hermano policonsumidor, dicha probabilidad aumenta entre 1.92 y 5.67 en comparación con los que no tienen hermanos policonsumidores y si su pareja es policonsumidora esta probabilidad aumenta entre 2.33 y 7.14 veces en comparación con aquéllos que tienen parejas no policonsumidoras. El modelo se ajusta bien $\left(\chi^{2}=123.05\right.$; $p<0.001)$, explica más de un $40 \%$ de la variabilidad del policonsumo $\left(R^{2}\right.$ de Nagelkerke $\left.=0.41\right)$ y clasifica correctamente a un $76.3 \%$ de los participantes.

Tabla 3

Resultados de la regresión logística binaria para predecir el policonsumo de los participantes ( $n=342)$.

\begin{tabular}{lcccc}
\hline Variable & B & 0.R. & IC 95\% & $p$ \\
\hline Sexo & -0.09 & 0.91 & $0.53: 1.57$ & 0.74 \\
Edad & 0.05 & 1.05 & $0.97: 1.14$ & 0.26 \\
$\begin{array}{l}\text { Policonsumo del mejor } \\
\text { amigo }\end{array}$ & 1.81 & 6.13 & $3.29: 11.43$ & $<0.001$ \\
$\begin{array}{l}\text { Policonsumo del padre } \\
\text { Policonsumo de la madre }\end{array}$ & 0.12 & 1.12 & $0.62: 2.02$ & 0.70 \\
$\begin{array}{l}\text { Policonsumo de algún } \\
\text { hermano }\end{array}$ & 1.19 & 3.30 & $1.92: 5.67$ & $<0.001$ \\
Policonsumo de la pareja & 1.41 & 4.08 & $2.33: 7.14$ & $<0.001$ \\
\hline
\end{tabular}

\section{Discusión}

El presente estudio ha permitido profundizar en el conocimiento de las conductas de consumo drogas de estudiantes universitarios españoles del ámbito de la salud y el deporte. Para ello, además de aportar resultados relativos a la frecuencia del consumo de sustancias legales e ilegales, se describen las características centrales del policonsumo y su relación con el contexto familiar y social en jóvenes universitario.

En primer lugar, uno de los principales intereses de este estudio radica en conocer la asociación del uso de más de una sustancia con el policonsumo de las personas de su entorno más próximo (padre o tutor, madre o tutora, hermanos/as, mejor amigo/a, y la pareja). Se halla una elevada concordancia entre el policonsumo de sustancias de los participantes y el de sus referentes más próximos, de modo que si el referente es policonsumidor es más probable que el participante también lo sea. Asimismo, el policonsumo 
de drogas de su mejor amigo, de algún hermano/a y de la pareja son las variables que mejor pronostican el uso de más de una sustancia entre los jóvenes. De acuerdo a la teoría del aprendizaje social considerada al inicio de este estudio, el consumo de sustancias es conceptualizado como una conducta intencional y socialmente aprendida a través de un proceso de modelado y reforzamiento, además de la interacción de factores personales y socioambientales. Así, la exposición repetida a modelos que utilizan sustancias influye directamente en la conducta de los jóvenes. Sin embargo, en este estudio el policonsumo de los padres deja de tener relevancia para predecir el policonsumo de sustancias en los hijos cuando se tiene en cuenta el policonsumo de otros referentes de edades similares como los amigos, los hermanos o la pareja. En este sentido, cabe resaltar dos aspectos de interés de acuerdo a dicho resultado. Por un lado, siguiendo con la teoría de Bandura (1987) las influencias del modelo están determinadas por factores que facilitan el efecto de modelado. Por ejemplo, la similitud con el modelo en relación a factores como la edad y/o el sexo, el atractivo o el interés con el que se evalúa el modelo, los vínculos emocionales que pueda comportar la relación, el nivel de interacción habitual con el modelo, o la posición social del modelo. Por otro lado, se hace hincapié en las características que presenta la etapa de la juventud junto con la experiencia particular de la vida universitaria. El mundo universitario ofrece al joven una etapa de crecimiento personal, expectativas, proyectos profesionales y nuevos retos. Muchos de estos estudiantes cambian su lugar de residencia y comienzan a establecer nuevos contactos con sus iguales, y con personas de distinto sexo, lo que implica un proceso de adaptación al nuevo entorno social. El policonsumo se da principalmente en contextos recreativos (ej. fiestas raves) con otros jóvenes donde el uso de drogas puede suponer un mayor riesgo para la salud (Fernández-Calderón, Lozano-Rojas y Rojas-Tejada, 2013). En este contexto los padres, que durante la etapa adolescente tienen un papel único como modelos de referencia primarios (Ferrer y Ayneto, 1991), disminuyen su influencia (Halley et al., 2014). Es decir, a medida que el joven crece y consigue una mayor independencia la influencia de apego de los padres hacia los hijos va perdiendo fuerza (Becoña, Fernández, Calafat y Fernández-Hermida, 2014). Finalmente, la edad y el sexo no predicen el uso de más de dos sustancias de los jóvenes cuando no se diferencian los patrones de policonsumo. La falta de asociación de las diferencias de sexo se encuentra apoyada por otros autores que ponen de manifiesto la importancia de considerar el policonsumo de tabaco y cannabis de igual modo para hombres y mujeres (Ramo et al., 2012). Sin embargo, otros trabajos encuentran diferencias según el sexo en el policonsumo de sustancias (Substance Abuse and Mental Health Services Administration, 2009; Whitehorne-Smith et al., 2012). Respecto a la edad, los resultados de este estudio no son consistentes con las diferen- cias halladas por otros autores (OEDT, 2011; Ramo et al., 2012). Por tanto, sería necesario un mayor número de investigaciones con el fin de aclarar las posibles inconsistencias respecto a las variables sociodemográficas referentes al sexo y la edad.

Otro objetivo consiste en conocer la frecuencia del consumo de drogas en una muestra de jóvenes universitarios españoles según el sexo. Así, la sustancia legal más consumida a diario es el tabaco. No obstante, pocos estudiantes informaron no consumir nunca alcohol (93.9\% son consumidores de alcohol). Los mayores porcentajes en consumo ocasional y alguna vez por semana pertenecen a los consumidores de alcohol. Estos datos se encuentran en consonancia con el último informe del OEDT (2011) en jóvenes de 15 a 34 años. Entre las drogas ilegales, destaca el cannabis como la primera sustancia más consumida. Los datos sobre consumo diario de cannabis superan a los hallados por el OEDT (2011) aunque sí se encuentran en la línea respecto al incremento del consumo desde el año 2007 hasta la actualidad, y con los resultados hallados en otros estudios (Font-Mayolas et al., 2006; Viña y Herrero, 2004). Además, el cannabis es la única sustancia que mostró diferencias en función del sexo con más consumidores varones que mujeres tal como indican otras investigaciones (Font-Mayolas et al., 2006; OEDT, 2011). Respecto al resto de sustancias, la cocaína es la sustancia ilegal más consumida por los universitarios después del cannabis. Este dato no es extraño dado que España es el país europeo, junto con Reino Unido, que presenta las tasas más elevadas de prevalencia de consumo de cocaína (OEDT, 2011). Finalmente, el Spice es la sustancia menos usada entre los estudiantes de la muestra. No obstante, merece especial atención la participación en el consumo de Spice. Aunque los porcentajes son inferiores a los hallados en adolescentes (OEDT, 2011), la frecuencia de consumo de uno de los usuarios es diaria y en todos los casos por el sexo masculino. El Spice es una mezcla de hierbas para fumar con efectos similares a los del cannabis de la que todavía se sabe poco (Mustata et al., 2009). Una de las primeras investigaciones realizadas sobre esta sustancia con una muestra amplia de estudiantes universitarios $(n=2.349)$ indica que ser hombre y consumidor de otras sustancias incrementa la probabilidad de consumir estos cannabinoides sintéticos (Stogner y Miller, 2014). No obstante, todavía son escasos los estudios realizados en ámbito español y en población universitaria. Se requiere mayor investigación sobre este tipo de droga emergente tanto en la juventud como en etapas anteriores prestando atención a los patrones de consumo y a los aspectos demográficos.

Un último objetivo de este estudio reside en describir los patrones de policonsumo de los jóvenes. Cerca de la mitad de la muestra es policonsumidora de dos o más sustancias. De éstos, un 29.4\% correspondían al Patrón A (alcohol + tabaco), un 50.7\% al Patrón B (cannabis + alcohol y/o taba- 
co), un $16.7 \%$ al Patrón C (cannabis + alcohol y/o tabaco + otra droga ilegal) y un $3.2 \%$ a otros patrones de policonsumo (alcohol + cocaína; tabaco + alcohol + cocaína; alcohol + inhalantes; anfetaminas + alucinógenos + Spice). Estos resultados se encuentran en la línea de la literatura científica registrando una relación positiva entre alcohol y tabaco como drogas mayoritarias, combinadas con otras sustancias como cannabis (Barret, Darredeau y Pihl, 2006; McCabe, Cranford, Morales y Young, 2006; O'Reilly y Jessen, 2005; Riquelme et al., 2012). De acuerdo con estos datos, el policonsumo de tabaco y cannabis también se reconoce por su relevancia (Burns, Ivers, Lindorff y Clough, 2000; Calafat et al., 2000) siendo una de las combinaciones más usadas por adolescentes y jóvenes adultos (Barrett et al., 2006; Ramo et al., 2012). Además, el patrón A es más común en las mujeres, el patrón $\mathrm{C}$ en los hombres y el patrón $\mathrm{B}$ no presenta diferencias por sexo. Una explicación plausible a estas diferencias podría hallarse atendiendo a los datos del OEDT (2011). En el caso de las mujeres, el tipo de consumo de determinadas sustancias en los últimos años, por ejemplo el consumo intensivo de alcohol o el consumo diario de tabaco, ha sufrido un incremento que ha permitido igualar su consumo al de los hombres. Respecto a los hombres, la extensión del uso de todas las sustancias es mayor que en las mujeres, especialmente las ilegales.

El presente estudio muestra algunas limitaciones. Dada la naturaleza transversal de este trabajo no puede evaluarse la dirección de las relaciones encontradas. Sería necesario completar este estudio con otros longitudinales observando posibles cambios a lo largo del tiempo. Otra limitación se refiere a la evaluación de las conductas de consumo de las personas cercanas al entorno de los participantes del estudio. Es decir, la evaluación de estas conductas se basa en las percepciones que tienen los jóvenes participantes. Igualmente, dado que las conductas de consumo de drogas pueden ser o no ser generalmente aceptadas por la sociedad, las respuestas de los participantes en el autoinforme podrían verse incrementadas o disminuidas por el posible efecto de la deseabilidad social. No obstante, aunque se intentó minimizar este sesgo garantizando la confidencialidad de las respuestas, sería aconsejable utilizar, además de autoinformes, otros procedimientos como por ejemplo escalas de valoración para los iguales. Estudios futuros deberían analizar si los mismos factores de riesgo que se asocian con el consumo de una única sustancia como tabaco, alcohol o cannabis, también podrían estar relacionados con el policonsumo de estas sustancias. Finalmente, en los últimos años el tipo de familia ha pasado de familias tradicionales (con padres biológicos) o extensas (convivencia con otros miembros de la familia) a familias monoparentales, reconstituidas por una nueva pareja e incluso con padres del mismo sexo (Becoña et al., 2012). Dada la importancia de estos cambios en la familia sería recomendable que próximos trabajos aborden la relación entre los nuevos tipos de familia y el policonsumo de sustancias.
A pesar de estas limitaciones, los resultados sugieren que el policonsumo de drogas en el entorno próximo de los jóvenes deviene uno de los elementos clave a tener en cuenta en futuras campañas preventivas del consumo de drogas.

\section{Reconocimientos}

Este trabajo ha sido posible gracias a la ayuda a la actividad investigadora de la Escola Universitària de la Salut i l'Esport (EUSES) en colaboración con la Universidad de Girona (UdG), año 2014.

\section{Conflicto de intereses}

Los autores declaran no tener conflicto de intereses.

\section{Referencias}

Bandura, A. (1987). Pensamiento y acción: Fundamentos sociales. Barcelona: Martínez Roca (Trabajo original publicado en 1986).

Bandura, A. y Walters, R.H. (1979). Aprendizaje social y desarrollo de la personalidad. Madrid: Alianza Universidad (Trabajo original publicado en 1959).

Barrett, S. P., Darredeau, C. y Pihl, R. O. (2006). Patterns of simultaneous polysubstance use in drug using university students. Human Psychopharmacology: Clinical and Experimental, 21, 255-263. doi: 10.1002/hup.766.

Becoña, E., Fernández, E., Calafat, A. y Fernández-Hermida, J. R. (2014). Apego y consumo de sustancias en la adolescencia: Una revisión de aspectos conceptuales y metodológicos. Adicciones, 26, 77-86.

Becoña, E., Martínez, U., Calafat, A., Juan, M., Duch, M. y Fernández-Hermida, J. R. (2012). ¿Cómo influye la desorganización familiar en el consumo de drogas de los hijos? Una revisión. Adicciones, 24, 253-268.

Burns, C. B., Ivers, R. G., Lindorff, K. J. y Clough, A. R. (2000). Cannabis: A Trojan horse for nicotine? Australian and New Zealand. Journal of Public Health, 24, 637637. doi: 10.1111/j.1467-842X.2000.tb00533.x.

Buu, A., Dipiazza, C.,Wang, J., Puttler, L. I., Fitzgerald, H. E. y Zucker, R. A. (2009). Parent, family, and neighborhood effects on the development of child substance use and other psychopathology from preschool to the start of adulthood. Journal of Studies on Alcohol and Drugs, 70, 489-498.

Calafat, A., Fernández, C., Becoña, E., Gil, E., Juan, M. y Torres, M. A. (2000). Consumo y consumidores de cannabis en la vida recreativa. Adicciones, 12, 197-230.

Connell, C. M., Gilreath, T. D., Aklin, W. M. y Brex, R. A. (2010). Social-ecological influences on patterns of substance use among non-metropolitan high school students. American Journal of Community Psychology, 45, 3648. doi: 10.1007/s10464-009-9289-x. 
Connor, J. P., Gullo, M. J., White, A. y Kelly, A. B. (2014). Polysubstance use: diagnostic challenges, patterns of use and health. Current Opinion in Psychiatry, 27, 269-275. doi: 10.1097/YCO.0000000000000069.

European Monitoring Centre for Drugs and Drug Addiction (2009a). Polydrug use: Patterns and responses. Luxembourg: Publications Office of the European Union. doi: $10.2810 / 26783$.

European Monitoring Centre for Drugs and Drug Addiction (2009b). Understanding the 'Spice' phenomenon. Luxembourg: Office for Official Publications of the European Communities. doi: 10.2810/27063.

European Monitoring Centre for Drugs and Drug Addiction (2011). Informe Anual 2011. El problema de la Drogodependencia en Europa. Luxembourg: Office for Official Publications of the European Communities. doi:10.2810/4450.

European Monitoring Centre for Drugs and Drug Addiction (2014). Informe Europeo sobre Drogas: Tendencias y Novedades. Luxembourg: Office for Official Publications of the European Communities. doi: 10.2810/32415.

Ferrer, X. y Ayneto, X. (1991). Nuevos métodos en la formación de padres para la prevención del abuso de drogas. Comunicación presentada en las XIX Jornadas Nacionales de Socidrogalcohol, Santa Cruz de Tenerife.

Fernández-Calderón, F., Lozano-Rojas, O. M. y Rojas-Tejada, A. J. (2013). Raves y consumo de drogas desde una perspectiva epidemiológica y psicosocial: una revisión bibliográfica sistemática. Adicciones, 25, 269-279.

Font-Mayolas, S., Gras, M. E., Cebrián, N., Salamó, A., Planes, M. y Sullman, M. J. (2013). Types of polydrug use among Spanish adolescents. Addictive Behaviors, 38, 1605-1609. doi: 10.1016/j.addbeh.2012.09.007.

Font-Mayolas, S., Gras, M. E. y Planes, M. (2006). Análisis del patrón de consumo de cannabis en estudiantes universitarios. Adicciones, 18, 337-344.

Font-Mayolas, S. y Planes, M. (2000). Efectos del modelado por parte de la familia, pareja y amigos en la conducta de fumar de jóvenes universitarios. Adicciones, 12, 467477.

Gómez, A., Herde, J., Laffee, A., Lobo, S. y Martín, E. (2007). Consumo de drogas lícitas e ilícitas por estudiantes universitarios. Facultad de Ingeniería. Universidad de Carabobo, 2006. Salus, 11, 41-45.

Halley, T. J., Forster, M., Wood, D., Baezconde-Garbanati, L. y Beth J. (2014). Problematic substance use among hispanic adolescents and young adults: implications for prevention efforts. Substance Use and Misuse, 49, 10251038. doi:10.3109/10826084.2013.852585.

Hawkins, J. D., Catalano, R. F. y Miller, J. Y. (1992). Risk and protective factors for alcohol and other drug problems in adolescence and early adulthood: Implications for substance-abuse prevention. Psychological Bulletin, 112, 64-105. doi: 10.1037//0033-2909.112.1.64.
Herrera, A., Simich, L., Strike, C., Brands, B., Giesbrecht, N. y Khenti, A. (2012). Policonsumo simultáneo de drogas en estudiantes de pregrado del área de la salud en una universidad, León-Nicaragua. Texto y Contexto Enfermagem, 21, 79-86. doi: 10.1590/S0104-07072012000500011.

Hughes, K., Bellis, M. A., Whelan, G., Calafat, A., Juan, M. y Blay, N. (2009). Alcohol, drogas, sexo y violencia: riesgos y consecuencias para la salud entre los jóvenes turistas británicos en las Islas Baleares. Adicciones, 21, 265-278.

Lores, A. P., Murcia, J. A. M., Gutiérrez, M. y Sicilia, A. (2003). La práctica físico-deportiva en la Universidad. Revista de Psicologia del Deporte, 12, 39-54.

Mariño, N., Castro, J. y Torrado, J. (2012). Funcionamiento ejecutivo en policonsumidores de sustancias psicoactivas. Revista de Psicología Universidad de Antioquia, 4, 49-63.

Martín-Montañez, E., Barón-López, F. J., Rubio, L. O., Pavía, J., Miranda, J. y Santos, I. M. (2011). Consumo de alcohol, tabaco, cannabis y otras sustancias psicoactivas en estudiantes de la Universidad de Málaga. Trastornos Adictivos, 13, 160-166. doi: 10.1016/S1575-0973(11)70032-0.

Martínez, I., Fuentes, M., García, F. y Madrid, I. (2013). El estilo de socialización familiar como factor de prevención o riesgo para el consumo de sustancias y otros problemas de conducta en los adolescentes españoles. Adicciones, 25, 235242.

Mason, W. A., Kosterman, R., Haggerty, K. P., Hawkins, J. D., Redmond, C., Spoth, R. L. y Shin, C. (2009). Gender moderation and social developmental mediation of the effect of a family-focused substance use preventive intervention on young adult alcohol abuse. Addictive Behaviors, 34, 599-605. doi: 10.1016/j.addbeh.2009.03.032.

McCabe, S. E., Cranford, J. A, Morales, M. y Young, A. (2006). Simultaneous and concurrent poly-drug use of alcohol and prescription drugs: prevalence, correlates, and consequences. Journal of Studies on Alcohol, 67, 529537.

Mustata, C., Torrens, M., Pardo, R., Pérez, C., The Psychonaut Web Mapping Group y Farré, M. (2009). Spice drugs: los cannabinoides como nuevas drogas de diseño. Adicciones, 21, 181-186.

Observatorio Español de la Droga y las Toxicomanías (2011). Informe 2011. Situación y tendencias de los problemas de drogas en España. Madrid: Ministerio de Sanidad, Política Social e Igualdad. Recuperado de: http://www. pnsd.msc.es/Categoria2/observa/pdf/oed2011.pdf.

Oliveira, E. B. y Furegato, A. R. F. (2008). El trabajo del estudiante de enfermería como un factor de riesgo para el consumo de alcohol y otras drogas. Revista Latinoamericana Enfermagem, 16, 565-571.

O'Reilly, B. y Jessen, J. (2005). Cannabis and other drug use by tertiary students in Darwin, Northern Territory, Australia. South Pacific. Journal of Psychology, 16, 18-29.

Patiño-Masó, J., Gras-Pérez, E., Font-Mayolas, S. y Baltasar-Bagué, A. (2013). Consumo de cocaína y poli- 
consumo de sustancias psicoactivas en jóvenes universitarios. Enfermería Clínica, 23, 62-67. doi: 10.1016/j. enfcli.2013.02.003.

Prieto, R., Simich, L., Strike, C., Brands, B., Giesbrecht, N. y Khenti, A. (2012). Diversity and complexity in the phenomenon of drugs: simultaneous polydrug use in university students in one university, Cundinamarca-Colombia. Texto y Contexto-Enfermagem, 21, 49-55. doi: 10.1590/ S0104-07072012000500006.

Ramo, D. E., Liu, H. y Prochaska, J. J. (2012). Tobacco and marijuana use among adolescents and young adults: A systematic review of their co-use. Clinical Psychology Review, 32, 105-121. doi: 10.1016/j.cpr.2011.12.002.

Riquelme, G., Simich, L., Strike, C., Brands, B., Girsbrecht, N. y Khenti, A. (2012). Características del policonsumo simultáneo de drogas en estudiantes de pregrado de carreras de ciencias de la salud de una universidad, Santiago-Chile. Texto y Contexto Enfermagem, 21, 34-40. doi: 10.1590/S0104-07072012000500004.

Stogner, J. M. y Miller, B. L. (2014). A spicy kind of high: a profile of synthetic cannabinoid users. Journal of Substance Use, 19, 199-205. doi:10.3109/14659891.2013.770 571.

Stone, A. L., Becker, L. G., Huber, A. M. y Catalano, R. F. (2012). Review of risk and protective factors of substance use and problem use in emerging adulthood. Addictive Behaviors, 37, 747-775. doi: 10.1016/j.addbeh.2012.02.014.

Substance Abuse and Mental Health Services Administration. (2009). Results from the 2008 National Survey on Drug Use and Health: National findings. Rockville, MD: Office of Applied Studies, NSDUH.

Tirado, R., Aguaded, J. I. y Marin, I. (2009). Factores de protección y de riesgo del consumo de alcohol en alumnos de la universidad de Huelva. Salud y Drogas, 9, 165183.

Trenz, R. C., Scherer, M., Harrell, P., Zur, J., Sinha, A. y Latimer, W. (2012). Early onset of drug and polysubstance use as predictors of injection drug use among adult drug users. Addictive Behaviors, 37, 367-372. doi: 10.1016/j.addbeh.2011.11.011.

Urrego, D. Z. (2002). Consumo de sustancias psicoactivas en estudiantes de especialidades médicas, Bogotá 2001. Revista de Salud Pública, 4, 59-73.

Varela, M., Salazar, I., Cáceres, D. y Tovar, J. (2007) . Consumo de sustancias psicoactivas ilegales en jóvenes: factores psicosociales asociados. Pensamiento Psicológico, 3 , $31-45$.

Vázquez, F. y Becoña, E. (2000). Factores de riesgo y escalada cannabinoide. Adicciones, 12 (Supl. 2), 175-184.

Veloza, M., Simich, L., Strike, C., Brands, B., Giesbrecht, N. y Khenti, A. (2012). Medio social y uso simultáneo de alcohol y tabaco en estudiantes universitarios de pregrado de carreras de ciencias de la salud de una universidad,
Cundinamarca-Colombia. Texto y Contexto Enfermagem, 21, 41-48. doi:10.1590/S0104-07072012000500005.

Viña, C. y Herrero, M. (2004). El consumo de sustancias psicoactivas en estudiantes de Psicología de la Universidad de la Laguna. International Journal of Clinical and Health Psychology, 4, 521-536.

Whitehorne-Smith, P., Simich, L., Strike, C., Brands, B., Giesbrecht, N. y Khenti, A. (2012). Gender differences in simultaneous polydrug use among undergraduate students of one university, Kingston-Jamaica. Texto y Contexto Enfermagem, 21, 74-78. doi: 10.1590/S010407072012000500010.

Wish, E. D., Fitzelle, D. B., O'Grady, K. E., Hsu, M. H. y Arria, A. M. (2006). Evidence for significant polydrug use among ecstasy-using college students. Journal of American College Health, 55, 99-104. doi:10.3200/JACH.55.2.99-104 